\title{
Exopolysaccharide Production in Response to Medium Acidification Is Correlated With an Increase in Competition for Nodule Occupancy
}

\author{
Barney A. Geddes, ${ }^{1}$ Juan E. González, ${ }^{2}$ and Ivan J. Oresnik ${ }^{1}$ \\ ${ }^{1}$ Department of Microbiology, University of Manitoba, Winnipeg, MB R3T 2N2, Canada; ${ }^{2}$ Department of Molecular and Cell \\ Biology, University of Texas at Dallas, Richardson, TX 75083-0688, U.S.A.
}

Submitted 29 June 2014. Accepted 21 July 2014.

\begin{abstract}
Sinorhizobium meliloti strains unable to utilize galactose as a sole carbon source, due to mutations in the De-Ley Doudoroff pathway $(\mathrm{dgoK})$, were previously shown to be more competitive for nodule occupancy. In this work, we show that strains carrying this mutation have galactose-dependent exopolysaccharide (EPS) phenotypes that were manifested as aberrant Calcofluor staining as well as decreased mucoidy when in an $\exp R^{+}$genetic background. The aberrant Calcofluor staining was correlated with changes in the $\mathrm{pH}$ of the growth medium. Strains carrying $d g o K$ mutations were subsequently demonstrated to show earlier acidification of their growth medium that was correlated with an increase expression of genes associated with succinoglycan biosynthesis as well as increased accumulation of high and low molecular weight EPS in the medium. In addition, it was shown that the acidification of the medium was dependent on the inability of $S$. meliloti strains to initiate the catabolism of galactose. To more fully understand why strains carrying the dgoK allele were more competitive for nodule occupancy, early nodulation phenotypes were investigated. It was found that strains carrying the $d g o K$ allele had a faster rate of nodulation. In addition, nodule competition experiments using genetic backgrounds unable to synthesize either succinoglycan or EPSII were consistent with the hypothesis that the increased competition phenotype was dependent upon the synthesis of succinoglycan. Fluorescent microscopy experiments on infected root-hair cells, using the acidotropic dye Lysotracker Red DND-99, provide evidence that the colonized curled root hair is an acidic compartment.
\end{abstract}

Collectively, the rhizobium-legume symbiosis contributes over 40 million tons of nitrogen per year into agricultural systems (Herridge et al. 2008). Enhancing the effectiveness of rhizobial inoculums is desirable, as it helps alleviate the requirement for synthetic nitrogen fertilizers that are economically and environmentally costly. One factor that limits the effectiveness of rhizobial inoculums is the inability to compete with indigenous rhizobia in the soil for nodule occupancy of legume hosts (Triplett and Sadowsky 1992).

Corresponding author: I. Oresnik; Telephone: +1.204.474.7587; Fax:+1.204.474.7603; E-mail: oresniki@cc.umanitoba.ca

* The $e$-Xtra logo stands for "electronic extra" and indicates that one supplementary figure is published online.

(C) 2014 The American Phytopathological Society
Sinorhizobium meliloti engages in nitrogen-fixing symbiosis with the agriculturally important forage Medicago sativa (alfalfa) and the model legume Medicago truncatula. Symbiosis between $S$. meliloti and its legume hosts begins with a signal exchange in the rhizosphere. S. meliloti produces Nod factor (NF) in response to flavonoids secreted by the legume (Spaink 2000). NF recognition by the legume host activates a complex signaling pathway that leads to root-hair curling and nodule development. Root-hair curling traps S. meliloti that have colonized the root hair within an apoplastic compartment called the curled colonized root hair (CCRH) (Oldroyd et al. 2011). Intracellular invasion of the root hair and subsequent cell layers proceeds by the formation of a plant-derived infection thread (IT) (Oldroyd et al. 2011). Rhizobia are eventually endocytosed by inner cortical cells and acquire a plant-derived symbiotic membrane before differentiating into nitrogen-fixing bacteroids (Oldroyd et al. 2011). Successful penetration of the IT requires both the continued biosynthesis of NF and the synthesis of symbiotic exopolysaccharides (EPS) (Jones et al. 2007).

$S$. meliloti produces two symbiotic types of EPS: succinoglycan and EPSII. Succinoglycan is a polymer of octasaccharide repeating units composed of seven glucose and one galactose residue and decorated with succinyl, pyruvyl, and acetyl modifications (Reinhold et al. 1994). EPSII is a polymer of a disaccharide repeating unit composed of an acetylated glucose and pyruvylated galactose residue (Her et al. 1990). The ability to synthesize one of these types of EPS is required for invasion of M. sativa by S. meliloti (Cheng and Walker 1998a; Glazebrook and Walker 1989; Leigh et al. 1985). Succinoglycan and EPSII are both synthesized in high and low molecular weight (HMW and LMW) fractions. The LMW fractions of both succinoglycan and EPSII have been implicated as being particularly important for symbiosis (Battisti et al. 1992; González et al. 1996). Despite the extensive study of the genetics and regulation of symbiotic EPS, their role in symbiosis remains unclear. Studies have suggested that they may play a role in dampening the legume immune response during invasion (Jones et al. 2008) or protection from reactive oxygen species encountered during invasion (Lehman and Long 2013).

The regulation of EPS synthesis is influenced by a number of environmental factors, including nitrogen and phosphate limitation, metal ions, salt, osmolarity, and $\mathrm{pH}$ (Breedveld et al. 1990; Dilworth et al. 1999; Hellweg et al. 2009; Leigh et al. 1985; Mendrygal and González 2000; Miller-Williams et al. 2006; Oresnik et al. 1994; Rossbach et al. 2008). The regulation of succinoglycan and EPSII production is controlled by several regulatory systems in S. meliloti, including ExoS/ChvI, SyrA/SyrM, and SinI/ExpR (Cheng and Walker 1998b; Glenn 
and Dilworth 1981; Marketon et al. 2003; Mulligan and Long 1989). The ExoS/ChvI two-component system activates succinoglycan biosynthesis and suppresses flagella biosynthesis in response to an unknown signal (Yao et al. 2004). The system is also inhibited by physical interaction of a periplasmic protein, ExoR, with the sensor kinase ExoS (Chen et al. 2008). $\mathrm{SinI} / \mathrm{ExpR}$ is a quorum-sensing system that regulates EPSII biosynthesis (Marketon et al. 2003). The wild-type S. meliloti Rm1021 contains an insertion element-disrupting $\exp R$ and, as a result, is unable to synthesize EPSII under normal conditions (Pellock et al. 2002).

In Bradyrhizobium japonicum, altered EPS production has been shown to provide a competitive advantage for nodule occupancy (Triplett and Sadowsky 1992). The ability to metabolize many different carbon sources has also been shown to influence competition for nodule occupancy in S. meliloti and Rhizobium leguminosarum (Ding et al. 2012; Fry et al. 2001; Jensen et al. 2005; Jiménez-Zurdo et al. 1995; Kohler et al. 2010; Oresnik et al. 1998; Vanderlinde et al. 2013; Yost et al. 2006). During the characterization of galactose catabolism in $S$. meliloti, we recently showed that a mutant that was unable to catabolize galactose had an increased ability to compete for nodule occupancy compared with the wild type (Geddes and Oresnik 2012). This phenotype was in contrast to the reduced ability to compete for nodule occupancy demonstrated in other carbon metabolism mutants (Ding et al. 2012; Fry et al. 2001; Jiménez-Zurdo et al. 1995; Kohler et al. 2010; Oresnik et al. 1998; Vanderlinde et al. 2013; Yost et al. 2006).

In this work, we describe further characterization of a galactose mutant of $S$. meliloti in pursuit of an explanation for increased competitiveness. Our data is consistent with the hypothesis that early succinoglycan production mediates increased competitiveness for nodule occupancy in galactose mutants of S. meliloti.

\section{RESULTS}

\section{Altered EPS production}

in a galactose mutant of $S$. meliloti.

S. meliloti Rm1021 carrying a mutation in the gene encoding 2-keto-3-deoxy-galactonokinase dgoK1 (SRmD144) had an increased ability to compete for nodule occupancy (Geddes and Oresnik 2012). This phenotype was of particular interest, as the inability to catabolize many other carbon sources results in a reduced (rather than increased) ability to compete for nodule occupancy (Ding et al. 2012; Fry et al. 2001; JiménezZurdo et al. 1995; Kohler et al. 2010; Oresnik et al. 1998; Vanderlinde et al. 2013; Yost et al. 2006). Since galactose is a subunit of both EPS produced by $S$. meliloti (succinoglycan and EPSII) and has been shown to be necessary for infection and nodule formation in Rm1021 (Leigh and Walker 1994), we screened for effects on EPS production.

Rm1021 and a transductant carrying the dgoK1 allele (SRmD304) were screened, using Calcofluor UV-fluorescence on various media containing galactose, for effects of the $d g o K$ mutation on succinoglycan. Strains were screened, in the presence or absence of galactose for effects on EPS production. The Calcofluor UV-fluorescence phenotypes associated with succinoglycan of Rm1021 were assessed visually. No differences were observed in the absence of galactose but, when galactose was present, the strain containing the $d g o K$ mutation showed a dull blue fluorescence in contrast to a bright blue-green fluorescence of the wild type when grown on rhizobium minimal medium (RMM) and yeast extract mannitol (YEM) or the absence of fluorescence on $1 / 2$ glutamate yeast extract mannitol (GYM) media (Table 1; Supplementary Fig. 1).

Strain Rm1021 is unable to synthesize EPSII under normal conditions because of a mutation in the response regulator $\exp R$ (Glazebrook and Walker 1989; Pellock et al. 2002). In order to assess effects on EPSII production as well as succinoglycan production, the $d g o K 1:: \operatorname{Tn} 5$ allele was transduced into an $\exp R^{+}$ variant of Rm1021, SRmA363, to make SRmD305. Differences in EPSII production in SRmA363 and SRmD305 were screened visually on the basis of colony mucoidy (Table 2).

No significant differences were observed when grown on any of the glycerol-containing media, but when galactose was present, SRmD305 was significantly less mucoid than was SRmA363 on RMM, YEM, and $1 / 2$ GYM. The Calcofluor fluorescence phenotypes in an $\exp R^{+}$background were consistent with those that were observed in Rm1021 and SRmD304 (Table 1).

Table 1. Calcofluor UV-fluorescence phenotypes of a galactose mutant

\begin{tabular}{|c|c|c|c|c|c|c|c|}
\hline \multirow[b]{2}{*}{ Strain } & \multirow[b]{2}{*}{ Genotype } & \multirow[b]{2}{*}{ Carbon source $^{\mathrm{a}}$} & \multicolumn{5}{|c|}{ Medium $^{\text {b }}$} \\
\hline & & & LB & VMM & RMM & YEM & 1/2 GYM \\
\hline Rm1021 & Wild-type & Glycerol & + & + & + & + & - \\
\hline Rm1021 & Wild-type & Galactose & + & + & + & + & - \\
\hline SRmD304 & dgoK1::Tn5 & Glycerol & + & + & + & + & - \\
\hline SRmD304 & dgoK1::Tn5 & Galactose & + & + & $+{ }^{\text {dull }}$ & $++^{\text {dull }}$ & $++^{\text {dull }}$ \\
\hline
\end{tabular}

Table 2. Colony mucoidy phenotypes of a galactose mutant in an $\exp R^{+}$background

\begin{tabular}{|c|c|c|c|c|c|c|c|}
\hline \multirow[b]{2}{*}{ Strain } & \multirow[b]{2}{*}{ Genotype } & \multirow[b]{2}{*}{ Carbon source ${ }^{\mathrm{a}}$} & \multicolumn{5}{|c|}{ Medium $^{\text {b }}$} \\
\hline & & & LB & VMM & RMM & YEM & 1/2 GYM \\
\hline SRmA363 & $\exp R^{+}$ & Glycerol & + & + & + & + & + \\
\hline SRmA363 & $\exp R^{+}$ & Galactose & + & + & + & + & + \\
\hline SRmD305 & $\exp R^{+}, d g o K 1:: \operatorname{Tn} 5$ & Glycerol & + & + & + & + & + \\
\hline SRmD305 & $\exp R^{+}, \operatorname{dgoK} 1:: \operatorname{Tn} 5$ & Galactose & + & + & - & - & - \\
\hline
\end{tabular}

${ }^{a}$ Galactose-containing medium also contained $15 \mathrm{mM}$ glycerol to support growth.

${ }^{\mathrm{b}} \mathrm{LB}=$ Luria Bertani, VMM = Vincent's minimal medium, RMM = rhizobium minimal medium, YEM = yeast extract mannitol, and GYM = glutamate yeast extract mannitol. Mucoid phenotypes: +, mucoid colony; -, nonmucoid colony. Phenotypes were recorded after 3 days of growth. 
Early EPS production and acidification of culture media by SRmD304.

The dull blue UV-fluorescence phenotype we observed was not consistent with previously observed phenotypes associated with succinoglycan production. To characterize EPS production in SRmD304, we wished to quantitatively assay EPS production in liquid medium over time. Since the EPS phenotypes we had observed were dependent on the presence of galactose in the culture media and galactose would interfere with the traditional anthrone-sulfuric acid method (Glazebrook and Walker 1989; Mendrygal and González 2000), we used an EPS precipitation-based method to quantify EPS (Marroqui et al. 2001; Yurgel et al. 2013).

The growth rates of both SRmD304 and Rm1021 were similar during the logarithmic growth phase and both attained a similar final optical density (Fig. 1A). Precipitable EPS from the cultures of SRmD304 and Rm1021 was detected at approximately 40 and $52 \mathrm{~h}$, respectively (Fig. 1C). Coincident with early EPS production, a sharp drop in $\mathrm{pH}$ was observed in the SRmD304 cultures (Fig. 1B).

Acidic $\mathrm{pH}$ has been previously shown to induce the expression of genes for $S$. meliloti succinoglycan biosynthesis (Hellweg et al. 2009). To determine whether the acidification of the medium by SRmD304 was responsible for the early EPS production, EPS was quantified in cultures of SRmD304 grown in the presence or absence of $50 \mathrm{mM}$ HEPES as a buffering agent. After $48 \mathrm{~h}$ of growth, the $\mathrm{pH}$ of the unbuffered culture was $4.95 \pm 0.01$, and $45.1 \pm 2.5 \mathrm{mg}$ of EPS per milligram of protein was precipitated, whereas the $\mathrm{pH}$ of the buffered culture was $6.36 \pm 0.03$ and no perceptible EPS was detected.

To address if acidification of growth medium may explain the observed Calcofluor phenotypes, various culture media were buffered with HEPES to either $\mathrm{pH} 6.5$ or 6.0. While the biomass visible on the plate was similar, the typical bright blue-green fluorescence was observed only at $\mathrm{pH} 6.5$ (data not shown).

\section{Early EPS produced in SRmD304 is succinoglycan.}

Since the Calcofluor UV-fluorescence phenotypes were ambiguous, we wished to directly determine the molecular identity of the EPS that was precipitated at earlier timepoints. EPS was precipitated and purified from both SRmD304 and Rm1021 cultures grown for $44 \mathrm{~h}$ in RMM containing galactose. No significant amounts of precipitable EPS from the wild-type cultures was observed. High-performance liquid chromatography (HPLC) analysis of the EPS isolated from the SRmD304 culture showed two peaks that ran coincident with the monosaccharides glucose and galactose. Quantification of these peaks showed that glucose and galactose were present at a 7:1 ratio, consistent with the exclusive production of succinoglycan. EPS was also precipitated and purified from Rm1021 and SRmD304 that were grown for 6 days in RMM containing galactose. EPS purified from these cultures showed glucose/galactose ratios of 5.3:1 and 5.8:1, respectively, suggesting that the precipitated EPS was predominantly succinoglycan (data not shown).

\section{HMW and LMW fractions of succinoglycan are synthesized at early timepoints.}

Since LMW EPS has been shown to affect early events in symbiosis and $S$. meliloti that was unable to catabolize galactose was more competitive for nodule occupancy, we analyzed the molecular weight profile (HMW vs. LMW) of the EPS that was produced by SRmD304. Initial experiments that were carried out to determine the EPS profile involved precipitating, purifying, and separating the EPS by chromatography. Using these methods, we found that, in our hands, HMW EPS was being preferentially precipitated (data not shown).

To determine if LMW fractions of succinoglycan were also produced at early timepoints, cells from these timepoints were incubated with $\mathrm{D}-\left[\mathrm{U}_{-}{ }^{14} \mathrm{C}\right]$ glucose to allow direct observation of the molecular weight profile of the succinoglycan that was synthesized. The results showed accumulation of label into both HMW and LMW fractions of succinoglycan. Based on radioactive counts, a distribution of approximately $15 \% \mathrm{HMW}$ succinoglycan and $85 \%$ LMW succinoglycan was observed for SRmD304 (Fig. 2A) and a distribution of approximately 5\% HMW and 95\% LMW succinoglycan was observed for Rm1021 (Fig. 2B). These values are similar to those previously reported for wild-type S. meliloti (Glenn et al. 2007).

\section{Gene expression correlates}

with early succinoglycan production.

The composition and molecular weight distribution of early EPS production in SRmD304 suggested that we observed early succinoglycan biosynthesis in the galactose mutant. To address whether this was due to the increased expression of genes involved in succinoglycan biosynthesis, RNA was isolated from Rm1021 and SRmD304 grown under the conditions of early EPS synthesis and was analyzed by quantitative reverse transcription-polymerase chain reaction (RT-PCR).

Consistent with the early synthesis of succinoglycan in SRmD304, we observed increased expression of the succino-
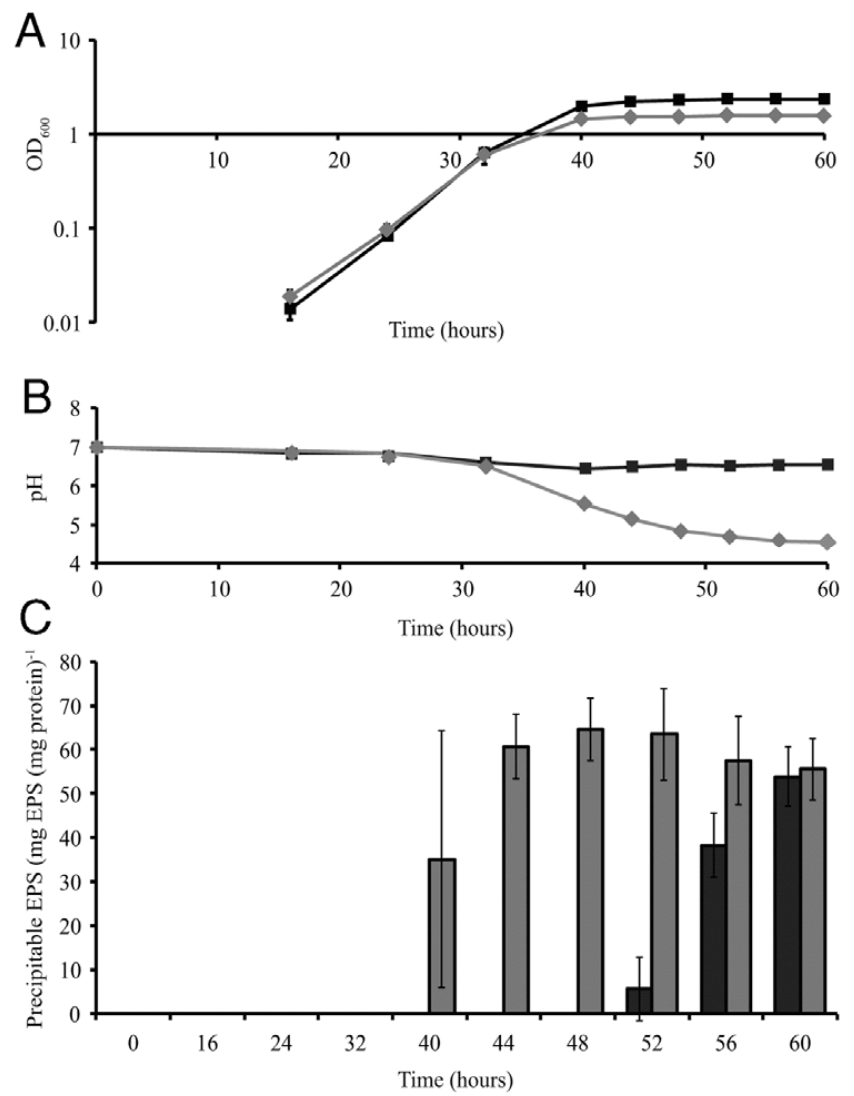

Fig. 1. Exopolysaccharide (EPS) production by Rm1021 and SRmD304. Black bars and black squares represent Rm1021. Gray bars and diamonds represent SRmD304. Strains were grown in rhizobium minimal medium with $15 \mathrm{mM}$ galactose. A, EPS was precipitated from $50 \mathrm{ml}$ of culture supernatant and was dried and weighed at regular intervals over $60 \mathrm{~h}$ of growth. $\mathbf{B}$, Optical density and $\mathbf{C}, \mathrm{pH}$ of cultures was monitored at each timepoint. Values represent the means \pm standard deviations of three independent biological replicates. When not seen, standard deviations are smaller than the symbol. 
glycan biosynthesis genes exo $Y$ and exoH and the endoglycanases responsible for generation of LMW fractions of succinoglycan exoK and exsH compared with Rm1021 (Table 3).

To investigate possible effects on other biologically relevant processes that may be associated with increased competition for nodule occupancy, representative genes of flagella $(f \lg B)$, cyclic $\beta$ - $(1,2)$ glucan $(n d v B)$, lipopolysaccharide (lps $B)$, EPSII (expE2), and NF biosynthesis (nodA) were tested for changes in gene expression. Whereas decreased expression of the flagella biosynthesis gene $f \lg B$ was observed, no significant changes in the expression of the other genes were observed (Table 3).

\section{SMc00588 encodes a galactose dehydrogenase that is required for galactose-dependent culture medium acidification.}

Galactose catabolism in S. meliloti uses the De Ley-Doudoroff pathway (Arias and Cerveñansky 1986). SRmD304 carries a mutation in the galactonokinase $d g o K 1$; thus, galactose catabolism is blocked at the step of the phosphorylation of 2keto-3-deoxy-galactonate in this mutant (Arias and Cerveñansky 1986; Geddes and Oresnik 2012). To determine if medium acidification was the result of a metabolic defect resulting from the build-up of intermediates, catabolism needed to be blocked such that the initial oxidation of galactose would not occur. We had previously isolated an insertion in galD (galD::
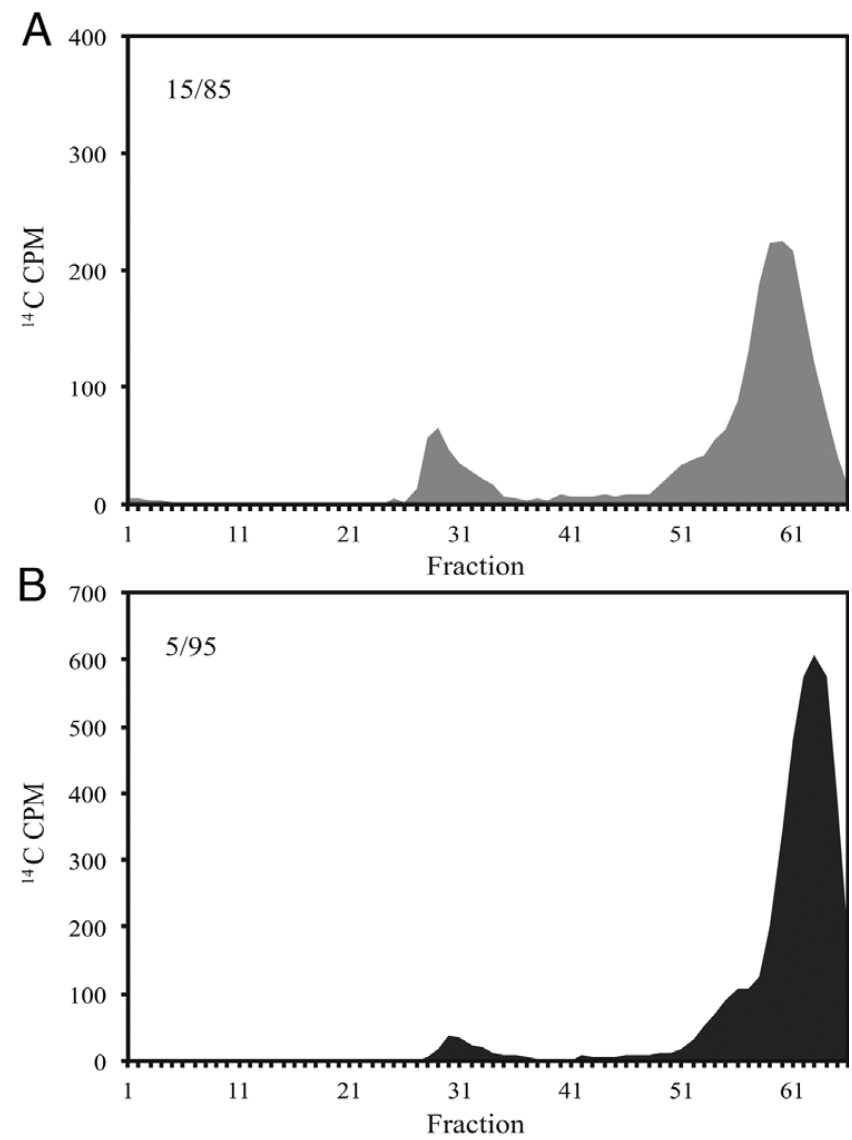

Fig. 2. Molecular-weight profile of exopolysaccharides (EPS) synthesized by $\mathrm{Rm} 1021$ and SRmD304. A, $\left[{ }^{14} \mathrm{C}\right]$-labeled succinoglycan from the supernatants of SRmD304 and $\mathbf{B}, \mathrm{Rm} 1021$ were isolated and were separated by column chromatography. The ratio of high moelcular weight (HMW) to low molecular weight (LMW) succinoglycan is indicated in the top left corner as a percentage. Fractions containing HMW and LMW succinoglycan were assigned based on previous experiments using the same column and matrix to separate radiolabeled succinoglycan (Glenn et al. 2007). The experiment was repeated twice showing consistent results.
Tn5-B20) that was polar on both $d g o K 1$ as well as $d g o A$. It was shown that, although a strain (SRmD441) carrying this allele was unable to utilize galactose as sole carbon source, it still contained a second enzyme that had galactose/arabinose dehydrogenase activity (Geddes and Oresnik 2012). Consistent with this, after $44 \mathrm{~h}$ of growth in medium containing galactose, medium acidification and EPS production were similar to that found in SRmD304 (data not shown).

Based on annotation and similarity to L-arabinose /galactose dehydrogenases identified in Agrobacterium tumefaciens and Azospirillum brasilense (Watanabe et al. 2006; Zhao and Binns 2011), we hypothesized that the putative galactose dehydrogenase $S M c 00588$ encoded for the redundant galactose dehydrogenase activity we had observed. To test this, we constructed a single crossover disruption in this gene, using the suicide vector pKNOCK-Gm. The resulting mutant, SRmD434 (SMc00588:: pKNOCK-Gm), was unable to grow using L-arabinose as a sole carbon source and grew slowly using galactose as a sole carbon source. Assays for galactose and L-arabinose dehydrogenase activities using nondenaturing polyacrylamide gel electrophoresis showed that SMc00588 is a galactose/L-arabinose dehydrogenase (data not shown). We note that during the characterization of L-arabinose catabolism in S. meliloti, an L-arabinose dehydrogenase was not identified (Poysti et al. 2007).

To block galactose catabolism at the first step, we constructed a double mutant of galD and SMc00588, yielding SRmD442. After $44 \mathrm{~h}$ of growth in RMM containing galactose and glycerol, SRmD442 showed wild-type $\mathrm{pH}$ levels and the absence of early EPS production, whereas SRmD441 (galD::Tn5-B20) showed medium acidification and early EPS production at levels similar to SRmD304, suggesting that the observed media acidification resulted from a metabolic defect dependent on the intracellular oxidation of galactose.

\section{Increased rate of early nodulation}

in the $S$. meliloti galactose mutant.

Increased competitiveness for nodule occupancy was previously observed in the $d g o K 1$ mutant SRmD144 (Geddes and Oresnik 2012). To ensure that the competitive phenotype was due to the Tn 5 insertion, the $d g o K 1$ allele was transduced into Rm1021 to yield SRmD304. Competition for nodule occupancy assays confirmed that the increased competition phenotype was transduced with the Tn5 insertion. Since competition for nodule occupancy is typically scored around 4 to 5 weeks after inoculation, we wondered if SRmD304 differed from the wild

Table 3. Changes in gene expression in SRmD304

\begin{tabular}{llc}
\hline Gene & \multicolumn{1}{c}{ Gene product function } & Gene expression $^{\mathbf{a}}$ \\
\hline exoY & Succinoglycan galactosyltransferase & $6.7^{\mathrm{b}}$ \\
exoH & Succinoglycan succinyltransferase & 7.8 \\
exoK & Succinoglycan endoglycanase & 6.0 \\
exsH & Succinoglycan endoglycanase & $7.8^{\mathrm{b}}$ \\
flgG & Flagellar basal-body rod protein & $0.15^{\mathrm{c}}$ \\
lpsB & LPS core biosynthesis & $\mathrm{NC}^{\mathrm{d}}$ \\
ndvB & B-(1,2) glucan transmembrane protein & $\mathrm{NC}$ \\
expE2 & EPSII glycosyltransferase & $\mathrm{NC}$ \\
nodA & Nod factor N-acyltransferase & $\mathrm{NC}$ \\
\hline
\end{tabular}

${ }^{a}$ Fold change in gene expression. Data expressed as $2^{\Delta \Delta \mathrm{Ct}}$ and represents fold expression in SRmD304 over Rm1021 grown in rhizobium minimal medium with $15 \mathrm{mM}$ galactose. The experiment also included SMc00128 as an internal control (Krol and Becker 2004). The table represents data from a single experiment. Unless otherwise noted, standard error between three independent experiments was within 1 cycle threshold (twofold change). The experiment was also repeated in triplicate using 1/2 glutamate yeast extract mannitol medium and showed consistent results.

b Data from two independent replicates.

${ }^{c} 0.15$ is equivalent to a 6.7 -fold reduction in gene expression.

${ }^{\mathrm{d}} \mathrm{NC}=$ no change. 
type with respect to any visible early nodulation characteristics, such as how quickly nodules formed.

The kinetics of nodule formation was monitored in over 100 alfalfa plants over five independent replicates in Rm1021 and SRmD304. Initial visible nodulation events occurred at 5 days postinoculation on plants inoculated with either Rm1021 or SRmD304 (Fig. 3A). Although the appearance of the first nodules occurred at the same time, the proportion of plants nodulated increased more rapidly for those inoculated with a strain carrying the $d g o K 1$ allele and attained $100 \%$ nodulation more quickly (Fig. 3A). This phenotype was most pronounced as $50 \%$ of the plants reached nodulation; $50 \%$ of plants inoculated with SRmD304 became nodulated by approximately 6 days postinoculation, whereas $50 \%$ of plants inoculated with $\mathrm{Rm} 1021$ became nodulated at approximately
8 days postinoculation (Fig. 3B). Taken together, these data suggest that the increased competitive phenotype that is observed after 4 weeks may be due to events that occur early during nodule formation.

\section{Competition for nodule occupancy is dependent upon succinoglycan.}

Increased production of succinoglycan has recently been correlated with enhanced symbiosis of S. meliloti 1021 with M. truncatula (Jones 2012), and galactose has been shown to be prevalent near the root surface of $M$. sativa seedlings (Bringhurst et al. 2001). We hypothesized that enhanced succinoglycan production during invasion may be mediating increased competitiveness for nodule occupancy in SRmD304. If the effects we had observed on EPS production in SRmD304
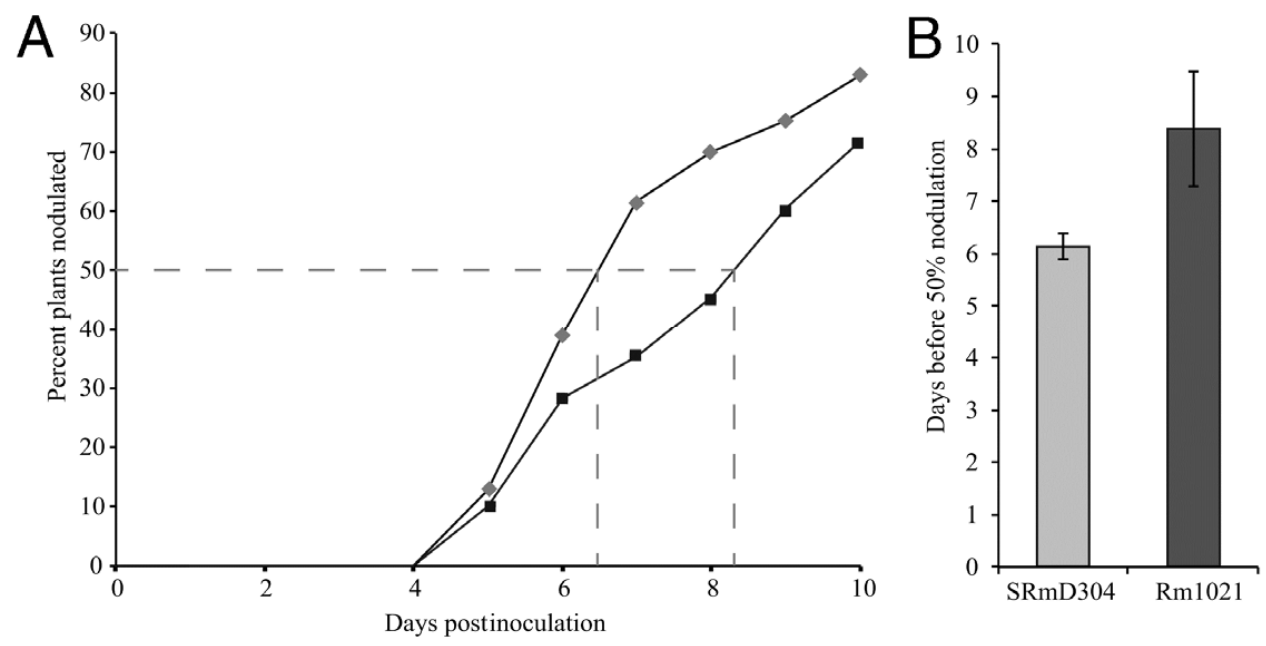

Fig. 3. Effects on nodule kinetics in a galactose mutant of Sinorhizobium meliloti. A, Percent of plants nodulated over time after inoculation with Rm1021 or SRmD304. Data is the sum of 100 plants over five independent replicates. The average standard deviation for the each data point is 11 and $7 \%$, respectively, for Rm1021 and SRmD304. B, Average number of days postinoculation before 50\% of plants became nodulated. The average and standard deviation was calculated from three independent replicates that contained at least 20 plants each. $P$ value $<0.0125$.
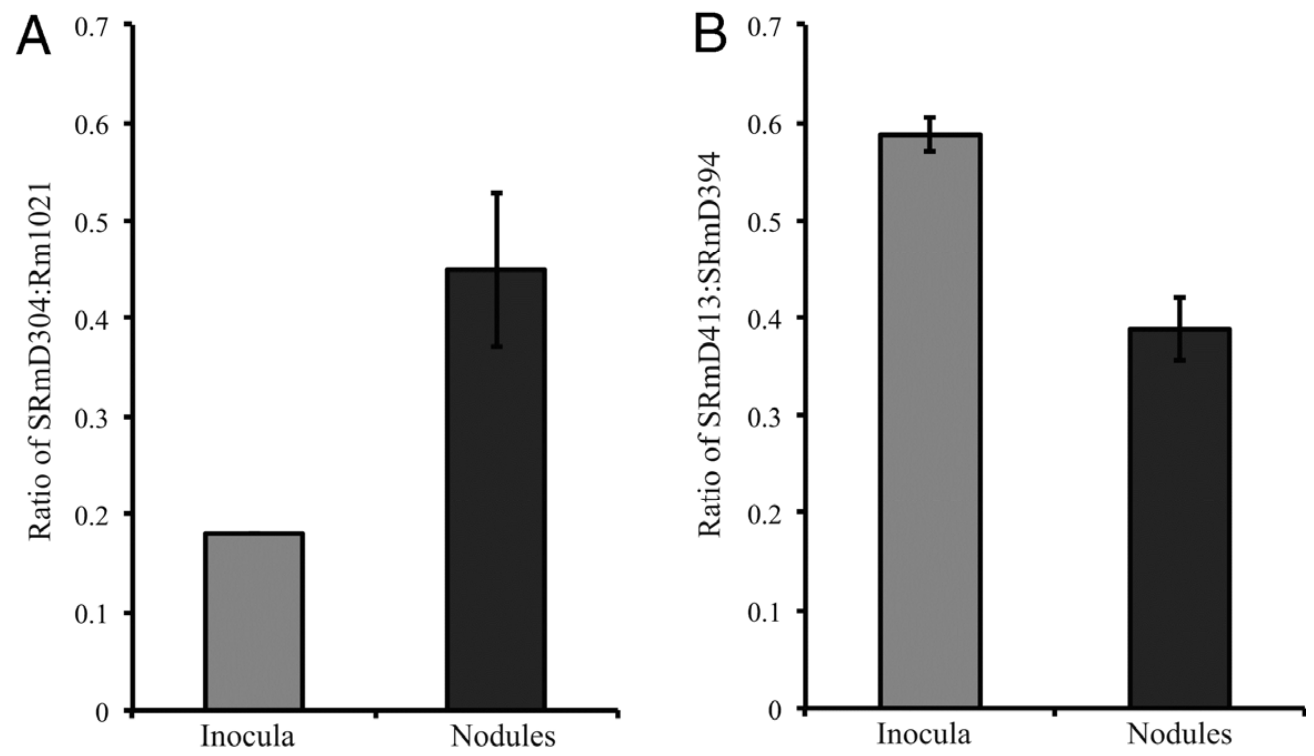

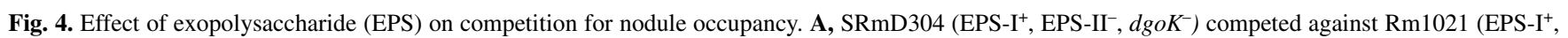
EPS-II ${ }^{-}, d g o K^{+}$) for nodule occupancy of Medicago sativa. The data are presented as the mean \pm standard deviation of the proportion of SRmD304 present in the inoculum (white bar) and isolated from nodules (dark gray bar). Data are the average of three independent replicates, each comprised of 30 plants. B, SRmD413 (EPS-I' ${ }^{-}$EPS-II' ${ }^{+}$, dgoK ${ }^{-}$) competed against SRmD394 (EPS-I', EPS-II ${ }^{+}$, dgoK ${ }^{+}$) for nodule occupancy (using EPSII for invasion) of $M$. sativa. The data are presented as the mean \pm standard deviation of the proportion of SRmD413 present in the inoculum (white bar) and isolated from nodules (dark gray bar). Data are the average of two independent replicates, each comprised of 30 plants. Reduced competitiveness of SRmD413 was also observed over three independent replicates using inoculum that contained a 1:5 ratio of SRmD413 to SRmD394. 
were occurring during the invasion of alfalfa, we predicted that a $S$. meliloti strain carrying a $d g o K 1$ mutation and using EPSII, rather than EPSI, to invade $M$. sativa should have a reduced ability to compete for nodule occupancy, as we observed reduced synthesis of EPSII in $\exp R^{+}$galactose mutants of $S$. meliloti (Table 2). Conversely, if the increased competitiveness was due to other phenomena, the competitive advantage would be maintained.

To carry out this experiment, we constructed a strain that was unable to synthesize succinoglycan in an $\exp R^{+}$background that is capable of EPSII synthesis, SRmD394 (exoY, expR $R^{+}$. SRmD394 was used in a competition-for-nodule-occupancy experiment with a galactose mutant with the same genetic background, SRmD413 (dgoK, exoY, expR ${ }^{+}$).

The results of the competition experiment show that, whereas mutants that used succinoglycan and contained the dgoK::Tn 5 allele were more competitive for nodule occupancy (Fig. 4A), those that relied solely on EPSII rather than succinoglycan to invade were significantly reduced in ability to compete for nodule occupancy (Fig. 4B). These data are consistent with the hypothesis that the in vitro effects on EPS production we have described in this work occur in planta, during the invasion of $M$. sativa by $S$. meliloti, and influence the ability of $S$. meliloti strains to compete for nodule occupancy.

\section{The CCRH defines an acidic compartment.}

Very little is known about the physiology of the CCRH. A number of studies have clearly shown that strains of $S$. meliloti unable to synthesize succinoglycan were compromised in their ability to extend IT from the CCRH (Cheng and Walker 1998a; Pellock et al. 2000). Based on the previous data, we hypothesized that exposure to an acidic environment during the invasion of $M$. sativa by $S$. meliloti may contribute to the activation of succinoglycan biosynthesis.

To address this, we utilized fluorescent microscopy using green fluorescent protein (GFP)-labeled $S$. meliloti as well as the acidotropic dye Lysotracker Red DND-99 to test for the possible acidic nature of the CCRH and IT. Based on its chemical properties Lysotracker Red DND-99 provides qualitative evidence of an acidic $\mathrm{pH}$ in a compartment in which its fluorescence co-localizes. Recently, these dyes have been used to demonstrate the acidic nature of the peribacteroid space in nitrogen fixing bacteroids in planta (Pierre et al. 2013).

Invasion events were visualized on plant roots inoculated with either Rm1021 or SRmD304 expressing GFP by fluorescent microscopy. Overall, several CCRH and IT were visible on most root systems. CCRH and IT occupied by S. meliloti were analyzed for co-localization of red fluorescence from Lysotracker Red DND-99. Co-localization of red fluorescence

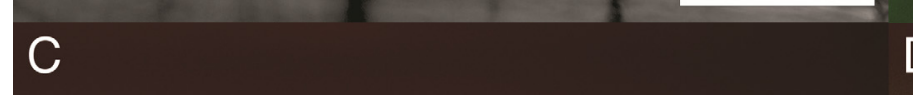

Fig. 5. The curled colonized root hair (CCRH) is an acidic compartment. Representative image of a CCRH 7 days after inoculation with S. meliloti strain SRmD304 expressing gfp and stained with Lysotracker Red DND-99. This experiment was replicated three times using completely independent biological material. A. Differential interference of contrast (DIC) image of CCRH. B. CCRH viewed using fluorescence of green fluorescent protein (GFP). C, CCRH viewed using red fluorescence of Lysotracker Red DND-99. D, Merged image showing the co-localization of green and red fluorescence of GFP and Lysotracker Red DND-99. 
with green fluorescence was observed in approximately $80 \%$ of CCRH (Fig. 5). Co-localization was seen in roots infected with either Rm1021 or SRmD304. Although we were able to visualize green fluorescent IT, we did not observe any colocalization of red fluorescence in the IT. We do not know whether this is due to a lack of penetration of Lysotracker Red 88 into the tissue or if it is due to the $\mathrm{pH}$ of the IT. However, co-localization of red fluorescence with the green fluorescence of $S$. meliloti strains in CCRH of M. sativa is consistent with the $\mathrm{CCRH}$ having an acidic nature.

\section{DISCUSSION}

In this work, we have shown that the galactose mutant SRmD304 produces succinoglycan at earlier timepoints than the wild type in media containing galactose (Fig. 1). We have also demonstrated the suppression of EPSII synthesis under similar conditions in backgrounds that contained an intact copy of the $\exp R^{+}$allele (Table 2) and that the observed EPS phenotypes correlated with competitiveness for nodule occupancy in galactose mutants (Figs. 3 and 4). In addition, we have also provided evidence that the $\mathrm{CCRH}$ may be an acidic compartment (Fig. 5).

The observed early synthesis of succinoglycan was dependent on the ability of SRmD304 to acidify its culture medium. The induction of genes associated with succinoglycan biosynthesis following a transition to growth in the presence of acidic $\mathrm{pH}$ levels has previously been reported (Hellweg et al. 2009). Consistent with this study, we observed the induction of genes for succinoglycan biosynthesis and the repression of genes associated with flagella biosynthesis in the acidic SRmD304 cultures. These data are consistent with the hypothesis that the acidification of its environment by SRmD304 resulted in the induction of genes for succinoglycan biosynthesis and the early production of succinoglycan.

We set out to identify possible explanations for the increased competitiveness for nodule occupancy we had previously observed in an S. meliloti galactose mutant (Geddes and Oresnik 2012). Three hypotheses can be generated regarding the phenotypes we observed in vitro and the increased competitiveness for nodule occupancy in SRmD304: i) early EPS production and medium acidification in vitro are unrelated to increased competiveness; ii) increased competitiveness results from environment acidification that occurs during invasion but is independent of effects on EPS production; and iii) increased competitiveness is mediated by the effects on EPS production caused by acidification of its environment by SRmD304 during invasion. We believe that several lines of evidence taken together favor the last hypothesis. Galactose containing compounds have been shown to be prevalent on the surface of alfalfa roots in sterilized and unsterilized soils (Bringhurst et al. 2001). Enhanced production of succinoglycan has recently been shown to enhance symbiosis of $S$. meliloti with $M$. truncatula (Jones 2012). The competitiveness for nodule occupancy of galactose mutants in this study correlated with effects on EPS production we observed in vitro. If increased competiveness for nodule occupancy was due to other factors in SRmD304, we would have expected a galactose mutant to maintain a competitive advantage over a strain that was unaffected in its ability to utilize galactose rather than succinoglycan when using EPSII to invade $M$. sativa. This was not observed.

The correlation between competitiveness and the ability to acidify the environment of $S$. meliloti in response to hexoses is present in another example in the literature. During growth using glucose as a carbon source, $S$. meliloti is able to acidify its environment by the periplasmic oxidation of glucose, using a membrane-bound pyrroloquinoline quinone (PQQ)-dependent glucose dehydrogenase (Bernardelli et al. 2001; Gosselin et al. 2001). Mutants of the gene encoding the PQQ-linked glucose dehydrogenase $g c d$ had a reduced ability to compete for nodule occupancy, despite being unimpaired in growth rate when using glucose as a sole carbon source (Bernardelli et al. 2001; Gosselin et al. 2001). Interestingly, gcd mutants also showed a delay in nodulation kinetics (Bernardelli et al. 2008), in contrast to the early nodulation we observed with plants inoculated with SRmD304.

A role for acidic $\mathrm{pH}$ in the interactions of microbes with eukaryotic hosts is not without precedent. The intracellular survival of Brucella spp. in human monocytes was found to be dependent on an acidic intraphagosomal $\mathrm{pH}$ (Rittig et al. 2001). Virulence genes in A. tumefaciens are activated by the two-component system VirA/VirG in cooperation with the sugar-binding protein ChvE in response to phenolic compounds, acidic pH, and hexoses (Charles and Nester 1993; He et al. 2009). Acid-inducible genes in A. tumefaciens are also regulated by a two-component system that is homologous to the ExoS/ChvI component system of $S$. meliloti, ChvG/ChvI (Li et al. 2002). A mechanism contributing to the induction of the ChvG/ChvI system by acidic $\mathrm{pH}$ levels has recently been demonstrated. Essentially the A. tumefaciens homolog of ExoR that inhibits ChvG in the periplasm is degraded under acidic conditions, allowing activation of the system and the induction of acid-inducible genes by ChvI (Wu et al. 2012). In $S$. meliloti, the ExoS/ChvI system has also been shown to be activated by the transient degradation of ExoR ( $\mathrm{Lu}$ et al. 2012). It is of note that SMc00588 (encoding a galactose dehydrogenase) has been shown to contain a ChvI-binding site (Bélanger and Charles 2013).

In this study, we have provided evidence that the CCRH may be an acidic compartment. It is possible that an acidic $\mathrm{pH}$ plays a role in the activation of succinoglycan biosynthesis in the CCRH in $S$. meliloti through the activation of the ExoS/ChvI system, as in A. tumefaciens. It is unclear whether the acidic environment of the CCRH is plant-derived, the result of the action of $S$. meliloti on its environment, or some combination of these events. It is also unclear whether the acidic nature of the CCRH is transient or persistent throughout invasion. Our working hypothesis is that the mutants unable to utilize galactose are more competitive for nodule occupancy because of an increased or earlier acidification of its environment in response to galactose in the $\mathrm{CCRH}$, resulting in enhanced succinoglycan production.

\section{MATERIALS AND METHODS}

\section{Bacterial strains, plasmids, and media.}

Bacterial strains and plasmids used and generated in this work are listed in Table 4. S. meliloti strains were grown routinely at $30^{\circ} \mathrm{C}$ on either Luria-Bertani (LB) or tryptone yeast extract (TY) as a complex medium (Sambrook et al. 1989). Vincent's minimal medium (VMM) (Vincent 1970), or RMM (Broughton et al. 1986), were used as a defined medium. Other media used include YEM (Vincent 1970) and $1 / 2$ GYM (Dylan et al. 1990). Carbon sources were filter-sterilized and were added to defined media to a final concentration of $15 \mathrm{mM}$ when relevant. To visualize succinoglycan production, $0.02 \%$ Calcofluor was added to media. Buffering of the media was accomplished using $50 \mathrm{mM}$ HEPES when relevant. When required, S. meliloti and Escherichia coli strains were grown in the following concentrations of antibiotic $(\mu \mathrm{g} / \mathrm{ml})$ : chloramphenicol, 20; gentamicin (Gm), 20 or 60; kanamycin, 20; neomycin, 200; streptomycin (Sm), 200; and tetracycline, 5. All antibiotics were filter-sterilized before use. 
DNA and genetic manipulations.

Conjugations and transductions were carried out essentially as previously described (Finan et al. 1984, 1988). Standard techniques were used for plasmid isolation, restriction enzyme digests, ligations, transformations, and agarose gel electrophoresis (Sambrook et al. 1989). To construct SRmD423, a 400-bp internal fragment of SMc00588 was amplified, restricted, and ligated into the suicide vector pKNOCK-Gm, creating pRW2. The plasmid was conjugated into $S$. meliloti Rm1021 and singlecrossover recombination events were selected for using $\mathrm{Sm}$ and Gm, as previously described (Richardson et al. 2004).

\section{Nondenaturing gel electrophoresis and dehydrogenase assays.}

Cells were grown in VMM medium with galactose or galactose and glycerol as sole carbon sources as previously described (Geddes and Oresnik 2012). Cell-free lysates were prepared and were separated using nondenaturing polyacrylamide gel electrophoresis, as previously described (Pickering and Oresnik 2008). Galactose dehydrogenase activity was detected in gels incubated either with or without $\mathrm{NAD}^{+} / \mathrm{NADP}^{+}$and galactose, using a $p$-nitroblue tetrazolium based dehydrogenase stain, as previously described (Geddes and Oresnik 2012; Latner and Skillen 1968).

Quantitative and composition analysis of EPS.

EPS precipitation and quantification was carried out as previously described (Marroquí et al. 2001; Yurgel et al. 2013). Characterization of the carbohydrate composition of EPS was done as previously described by Marketon and associates (2003), with some modifications. Rm1021 and SRmD304 were grown in RMM containing galactose (in addition to mannitol). EPS was isolated from SRmD304 after $44 \mathrm{~h}$ of growth and from both SRmD304 and Rm1021 after 6 days of growth. Briefly, 1 liter of cells was harvested by centrifugation, and EPS was precipitated and concentrated by lyophilization. Residual galactose from the medium was removed from the EPS by using a 1,000-molecular-weight-cutoff membrane (Spectrum) and dialyzing against water. A fraction of the dialyzed EPS solution was mixed with an equal volume of $4 \mathrm{M}$ trifluoroacetic acid and was hydrolyzed overnight at $100^{\circ} \mathrm{C}$ in a sealed glass vial. Analyses by high-pressure anion-exchange chromatography were performed on a Dionex DX 500 HPLC system with a CarboPac MA1 column, using a pulsed amperometric detector with a gold working electrode. The samples were eluted isocratically using a three-step protocol as follows: i) $500 \mathrm{mM} \mathrm{NaOH}$ for $20 \mathrm{~min}$, ii) a linear gradient from $500 \mathrm{mM}$ $\mathrm{NaOH}$ to $1 \mathrm{M}$ Na-acetate and $500 \mathrm{mM} \mathrm{NaOH}$ over $20 \mathrm{~min}$, and iii) $1 \mathrm{M} \mathrm{Na}$-acetate, $500 \mathrm{mM} \mathrm{NaOH}$ for $20 \mathrm{~min}$. The carbohydrate composition of the EPS was calculated as a glucose/ galactose ratio based on the elution times of known glucose and galactose standards.

\section{EPS molecular-weight profile.}

Analysis of the molecular-weight profile of EPS synthesized in Rm1021 and SRmD304 was performed as previously described with modifications (Glenn et al. 2007). Rm1021 and SRmD304 were first grown overnight in TY and, then, subcultured (1:200 dilution) into $25 \mathrm{ml}$ of RMM containing galactose (in addition to mannitol). Strains were then grown overnight at $30^{\circ} \mathrm{C}$ with constant shaking. The next day, cells were harvested by centrifugation $(13,000 \times g$ for $15 \mathrm{~min})$ at equivalent time-

Table 4. Bacterial strains and plasmids

\begin{tabular}{|c|c|c|}
\hline Strain or plasmid & Genotype or phenotype ${ }^{a}$ & Reference or source \\
\hline \multicolumn{3}{|c|}{ Sinorhizobium meliloti } \\
\hline Rm1021 & SU47 str-21, $\mathrm{Sm}^{\mathrm{R}}$ & T. M. Finan \\
\hline $\operatorname{Rm} 7055$ & Rm1021 exoY::Tn5, $\mathrm{Nm}^{\mathrm{R}}$ & Reuber and Walker 1993 \\
\hline RmH580 & Rm5000 gcd:: Tn5-233 & T. M. Finan \\
\hline SRmA363 & Rm1021 $\exp R^{+}$ & Miller-Williams et al. 2006 \\
\hline SRmD144 & $\mathrm{Rm} 1021$ dgoK1::Tn5, $\mathrm{Nm}^{\mathrm{R}}$ & Geddes and Oresnik 2012 \\
\hline SRmD288 & $\mathrm{Rm} 1021$ dgoK1::Tn5-233, $\mathrm{Gm}^{\mathrm{R}}, \mathrm{Sp}^{\mathrm{R}}$ & This work \\
\hline SRmD298 & Rm1021 galD::Tn5-B20, Nm ${ }^{\mathrm{R}}$ & Geddes and Oresnik 2012 \\
\hline SRmD304 & dgoK1::Tn5 $\Phi(\mathrm{SRmD} 144) \rightarrow \mathrm{Rm} 1021, \mathrm{Nm}^{\mathrm{R}}$ & This work \\
\hline SRmD305 & dgoK1::Tn5 $\Phi(\mathrm{SRmD} 144) \rightarrow \mathrm{SRmA} 363, \mathrm{Nm}^{\mathrm{R}}$ & This work \\
\hline SRmD394 & exoY::Tn5 $\Phi(\operatorname{Rm} 7055) \rightarrow \mathrm{SRmA} 363$ & This work \\
\hline SRmD413 & dgoK1::Tn5-233 Ф(SRmD288) $\rightarrow$ SRmD394, $\mathrm{Nm}^{\mathrm{R}}, \mathrm{Gm}^{\mathrm{R}}, \mathrm{Sp}^{\mathrm{R}}$ & This work \\
\hline SRmD427 & gcd::Tn5-233 Ф (RmH580) $\rightarrow$ Rm1021 & This work \\
\hline SRmD440 & gcd::Tn5-233 Ф (RmH580) $\rightarrow$ SRmD304 & This work \\
\hline SRmD434 & gal::pKNOCK-Gm, Gm ${ }^{\mathrm{R}}$ & This work \\
\hline SRmD441 & galD::Tn5-B20 Ф (SRmD298) $\rightarrow$ Rm1021, $\mathrm{Nm}^{\mathrm{R}}$ & This work \\
\hline SRmD442 & gal::pKNOCK-Gm $\Phi($ SRmD434) $\rightarrow$ SRmD441 & This work \\
\hline SRmD443 & gal::pKNOCK-Gm $\Phi($ SRmD434) $\rightarrow$ SRmD304 & This work \\
\hline \multicolumn{3}{|l|}{ Escherichia coli } \\
\hline DH5 $\alpha$ & $\Lambda^{-} \Phi 80 \mathrm{~d}$ lacZM15 (lacZYA-argF)U169 recAlendA1 hsdR17( $\left.\mathrm{r}_{\mathrm{K}}{ }^{-} \mathrm{m}_{\mathrm{K}}{ }^{-}\right)$supE44 thi-1 gyrA relA1 & Hanahan 1983 \\
\hline DH5 $\alpha \lambda$ pir & $\lambda$ pir lysogen of DH5 $\alpha$ & House et al. 2004 \\
\hline MM294A & pro-82-thi-1 hsdR17 supE44 & Finan et al. 1986 \\
\hline MT607 & MM294A recA56 & Finan et al. 1986 \\
\hline MT616 & MT607 (pRK600) & Finan et al. 1986 \\
\hline \multicolumn{3}{|l|}{ Plasmid } \\
\hline pAA10 & Galactose complementing cosmid, $\mathrm{Tc}^{\mathrm{R}}$ & Geddes and Oresnik 2012 \\
\hline pBG56 & $\mathrm{pCO} 36$ dgoK1, $\mathrm{Tc}^{\mathrm{R}}$ & Geddes and Oresnik 2012 \\
\hline pCO37 & pRK7813 containing $a t t B$ sites, Gateway® compatible destination vector & Jacob et al. 2008 \\
\hline pHC60 & $\mathrm{pHC} 41, \mathrm{~S} 65 \mathrm{~T} g f p$ & Cheng and Walker 1998b \\
\hline pPH1JI & IncP plasmid, $\mathrm{Gm}^{\mathrm{R}}$ & Beringer et al. 1978 \\
\hline pKNOCK-Gm & Suicide vector, $\mathrm{Gm}^{\mathrm{R}}$ & Alexeyev 1999 \\
\hline pRK600 & pRK2013 nptI::Tn9, Cm ${ }^{\mathrm{R}}$ & Finan et al. 1986 \\
\hline pRK607 & pRK2013::Tn5-233 & De Vos et al. 1986 \\
\hline pRK7813 & Broad-host-range vector, $\mathrm{Tc}^{\mathrm{R}}$ & Jones and Gutterson 1987 \\
\hline pRW2 & gal internal fragment in $\mathrm{pKNOCK}-\mathrm{Gm}, \mathrm{Gm}^{\mathrm{R}}$ & This work \\
\hline
\end{tabular}


points, when they reached $\mathrm{pH}$ levels of approximately 5.75 and 6.5 , respectively, for SRmD304 and Rm1021. Cell pellets were washed with $\mathrm{RMM}$ salts adjusted to the $\mathrm{pH}$ of the culture medium and were resuspended in $1 \mathrm{ml}$ of RMM. These cell suspensions were incubated with $6 \mu \mathrm{Ci}$ of $\mathrm{D}-\left[\mathrm{U}_{-}{ }^{14} \mathrm{C}\right]$ glucose for $4 \mathrm{~h}$ with constant shaking at $30^{\circ} \mathrm{C}$. Following incubation, samples were boiled for $5 \mathrm{~min}$, were placed on ice for $15 \mathrm{~min}$. and were pelleted $(15,000 \times g$ for $15 \mathrm{~min}$.). The resultant supernatant was dialyzed against $500 \mathrm{ml}$ of water using a 1,000molecular-weight-cutoff Pur-A-Lyzer dialysis kit (Sigma). Dialysis was performed over 3 days, with water changed every $12 \mathrm{~h}$. Succinoglycan-containing supernatant $(300 \mu \mathrm{l})$ was fractionated by column chromatography $(1 \mathrm{~cm} \times 100 \mathrm{~cm})$ using Bio-Gel P-6 (Bio-Rad). Preequilibration and elution was performed using a pyridinium acetate buffer (0.1 M, pH 5.0) (González et al. 1998). Fractions were collected in 1-ml aliquots, and radioactivity was detected by liquid scintillation with a Beckman LS6500 scintillation counter. Fractions were corrected for background $\mathrm{D}-\left[\mathrm{U}-{ }^{14} \mathrm{C}\right]$ glucose monomers. Determination of fractions containing HMW and LMW succinoglycan were based on previously reported distributions in $S$. meliloti, using identical column chromatography conditions (Glenn et al. 2007).

RNA isolation, cDNA synthesis, and quantitative RT-PCR.

For RNA isolation, bacterial cultures were grown as described above. Cells were harvested by centrifugation and cell pellets were frozen immediately in liquid nitrogen. RNA was then isolated using the Qiagen RNAeasy kit as previously described (Geddes et al. 2010). Approximately $200 \mathrm{ng}$ of cDNA sample was used as a template for quantitative RT-PCR. Reactions were performed using the SYBR green RT-PCR kit from Invitrogen as recommended by the manufacturer. The RT-PCR reaction was performed as previously described (Geddes et al. 2010). The RT-PCR primers used to measure EPS and flagella gene expression have been previously described (Glenn et al. 2007; Hoang et al. 2008). Other RT-PCR primers used are as follows: $l p s B$ forward: CATACCGGATGGAGCAAGTT, $\operatorname{lps} B$ reverse GAAGAGGTCGGTCCCTTTCT; $n d v B$ foreword: AC AACACCTCCATCGCACAG, $n d v B$ reverse: ATCGATCTTG CTGACGCTTT; and nodA forward: ACCACCAGGAGCTC TCAGAA, nodA reverse: GCGTATAAGCCCAGTTCAGC.

\section{Competition for nodule occupancy assays.}

Competition for nodule occupancy was assessed as previously described (Geddes et al. 2010). Briefly, alfalfa seeds were surface-sterilized and were germinated for $48 \mathrm{~h}$ in the dark on $1.5 \%$ agar. Seedlings (10 per jar) were then planted into sterile Leonard jar assemblies containing sand/vermiculite (1:1) that had been soaked with nitrogen-free Jensen's medium. Seedlings were inoculated after 2 days of growth with overnight cultures that were diluted 1:100 in sterile distilled water. Nodule occupancy was assessed after 30 days. At least 50 root nodules from each experiment were sterilized, and aliquots of each nodule extract were spotted onto LB plates containing antibiotics for strain differentiation. Competition was assessed by comparing the proportion of the mutant strain found in the inoculum with the proportion that was isolated from nodules. Significance was evaluated using a Student's paired $t$-test, assuming a $P$ value $<0.05$ indicated a significant difference in competitiveness.

\section{Nodule kinetics assays.}

Sterile alfalfa seedlings were planted in test-tube slants containing $10 \mathrm{ml}$ of Jensen's agar. After 2 days of growth, slants were inoculated with $100 \mu \mathrm{l}$ of strains grown and diluted in sterile water as described above. Nodule kinetics was assessed by counting the number of nodules formed on each plant daily over a 14-day period. Collectively, more than 100 plants over five independent replicates were monitored for nodule kinetics.

\section{Fluorescent microscopy.}

Plants were grown for fluorescent microscopy essentially as previously described with some modifications (Cheng and Walker 1998a; Gage et al. 1996). Sterile seedlings were placed onto microscope slides that contained $2 \mathrm{ml}$ of solidified Jensen's agar and were subsequently covered with dialysis membrane (14,000 molecular weight cutoff). Seedlings were inoculated as described above. These were incubated in 50-ml tubes containing $20 \mathrm{ml}$ of liquid Jensen's medium and were loosely covered with plastic cling wrap. Typical experiments contained at least 10 infected plants for each strain tested.

Visualization of CCRH and IT was performed 7 days postinoculation. To determine if CCRH were acidic, Lysotracker Red DND-99 was added to the Jensen's medium to a final concentration of $8 \mu \mathrm{M}$ before overnight incubation. The plants were destained in liquid Jensen's medium without the stain for at least $30 \mathrm{~min}$ before observation.

Roots were examined by fluorescent microscopy using a Zeiss Axio Imager.Z1 equipped with an AxioCamMR digital camera. Rhizobium strains expressing GFP were visualized using Zeiss filter set 38 (Endow GFP). Lysotracker Red DND99 fluorescence was observed using Zeiss filter set 20 (Rhodamine). Composite images were generated using Adobe Photoshop CS5.

\section{ACKNOWLEDGMENTS}

This work was funded by a Natural Sciences and Engineering Research Council of Canada (NSERC) Discovery grant to I. J. Oresnik, and National Science Foundation grant MCB-9733532 and National Institutes of Health grant 1R01GM069925 to J. E. González. B. A. Geddes acknowledges funding in the form of an NSERC Alexander Graham Bell Canada Graduate Scholarships-Doctoral Program award as well as an NSERC Michael Smith Foreign Study Supplement. The authors are grateful for technical assistance from R. Wong.

\section{LITERATURE CITED}

Alexeyev, M. 1999. The pKNOCK series of broad-host-range mobilizable suicide vectors for gene knockout and targeted DNA insertion into the chromosome of gram-negative bacteria. Biotechniques 26:824-828.

Arias, A., and Cerveñansky, C. 1986. Galactose metabolism in Rhizobium meliloti L5-30. J. Bacteriol. 167:1092-1094.

Battisti, L., Lara, J. C., and Leigh, J. A. 1992. Specific oligosaccharide form of the Rhizobium meliloti exopolysaccharide promotes nodule invasion in alfalfa. Proc. Natl. Acad. Sci. U.S.A. 89:5625-5629.

Bélanger, L., and Charles, T. C. 2013. Members of the Sinorhizobium meliloti ChvI regulon identified by a DNA binding screen. BMC Microbiol. 13:132.

Beringer, J. E., Beynon, J. L., Buchanan-Wollason, A. V., and Johnston, A. W. B. 1978. Transfer of the drug resistance transposon Tn5 to Rhizobium. Nature 276:633-634.

Bernardelli, C. E., Luna, M. F., Galar, M. L., and Boiardi, J. L. 2001. Periplasmic PQQ-dependent glucose oxidation in free-living and symbiotic rhizobia. Curr. Microbiol. 42:310-315.

Bernardelli, C. E., Luna, M. F., Galar, M. L., and Boiardi, J. L. 2008. Symbiotic phenotype of a membrane-bound glucose dehydrogenase mutant of Sinorhizobium meliloti. Plant Soil 313:217-225.

Breedveld, M., Zevenhuizen, L., and Zehnder, A. 1990. Osmotically induced oligo-and polysaccharide synthesis by Rhizobium meliloti SU-47. J. Gen. Appl. Microbiol. 136:2511-2519.

Bringhurst, R. M., Cardon, Z. G., and Gage, D. J. 2001. Galactosides in the rhizosphere: Utilization by Sinorhizobium meliloti and development as a biosensor. Proc. Natl. Acad. Sci. U.S.A. 98:4540-4545.

Broughton, W., Wong, C., Lewin, A., Samrey, U., Myint, H., Meyer, H., Dowling, D., and Simon, R. 1986. Identification of Rhizobium plasmid sequences involved in recognition of Psophocarpus, Vigna, and other legumes. J. Cell Biol. 102:1173-1182.

Charles, T. C., and Nester, E. W. 1993. A chromosomally encoded two- 
component sensory transduction system is required for virulence of Agrobacterium tumefaciens. J. Bacteriol. 175:6614-6625.

Chen, E. J., Sabio, E. A., and Long, S. R. 2008. The periplasmic regulator ExoR inhibits ExoS/ChvI two-component signalling in Sinorhizobium meliloti. Mol. Microbiol. 69:1290-1303.

Cheng, H.-P., and Walker, G. C. 1998a. Succinoglycan is required for initiation and elongation of infection threads during nodulation of alfalfa by Rhizobium meliloti. J. Bacteriol. 180:5183-5191.

Cheng, H.-P., and Walker, G. C. 1998b. Succinoglycan production by Rhizobium meliloti is regulated through the ExoS-ChvI two-component regulatory system. J. Bacteriol. 180:20-26.

De Vos, G. F., Walker, G. C., and Signer, E. R. 1986. Genetic manipulations in Rhizobium meliloti utilizing two new transposon Tn5 derivatives. Mol. Gen. Genet. 204:485-491.

Dilworth, M. J., Rynne, F. G., Castelli, J. M., Vivas-Marfisi, A. I., and Glenn, A. R. 1999. Survival and exopolysaccharide production in Sinorhizobium meliloti WSM419 are affected by calcium and low $\mathrm{pH}$ Microbiology 145:1585-1593.

Ding, H., Yip, C. B., Geddes, B. A., Oresnik, I. J., and Hynes, M. F. 2012 Glycerol utilization by Rhizobium leguminosarum requires an $\mathrm{ABC}$ transporter and affects competition for nodulation. Microbiology 158:1369-1378

Dylan, T., Helinski, D., and Ditta, G.S. 1990. Hypoosmotic adaptation in Rhizobium meliloti requires $\beta$-(1-2)-glucan. J. Bacteriol. 172:14001408.

Finan, T. M., Hartwieg, E., Lemieux, K., Bergman, K., Walker, G. C., and Signer, E. R. 1984. General transduction in Rhizobium meliloti. J. Bacteriol. 159:120-124.

Finan, T. M., Hirsch, A. M., Leigh, J. A., Johansen, E., Kuldau, G. A., Deegan, S., Walker, G. C., and Signer, E. R. 1985. Symbiotic mutants of Rhizobium meliloti that uncouple plant from bacterial differentiation. Cell 40:869-877.

Finan, T. M., Kunkel, B., de Vos, G. F., and Signer, E. R. 1986. Second symbiotic megaplasmid in Rhizobium meliloti carrying exopolysaccharide and thiamine synthesis genes. J. Bacteriol. 167: 66-72.

Finan, T. M., Oresnik, I., and Bottacin, A. 1988. Mutants of Rhizobium meliloti defective in succinate metabolism. J. Bacteriol. 170:3396-3403.

Fry, J., Wood, M., and Poole, P. S. 2001. Investigation of myo-inositol catabolism in Rhizobium leguminosarum bv. viciae and its effect on nodulation competitiveness. Mol. Plant-Microbe Interact. 14:10161025

Gage, D. J., Bobo, T., and Long, S. R. 1996. Use of green fluorescent protein to visualize the early events of symbiosis between Rhizobium meliloti and alfalfa (Medicago sativa). J. Bacteriol. 178:7159-7166.

Geddes, B. A., Pickering, B. S., Poysti, N. J., Yudistira, H., Collins, H., and Oresnik, I. J. 2010. A locus necessary for the transport and catabolism of erythritol in Sinorhizobium meliloti Microbiology 156:29702981

Geddes, G. A., and Oresnik, I. J. 2012. Inability to catabolize galactose leads to increased ability to compete for nodule occupancy in Sinorhizobium meliloti. J. Bacteriol. 194:5044-5505

Glazebrook, J., and Walker, G. C. 1989. A novel exopolysaccharide can function in place of the Calcofluor-binding exopolysaccharide in nodulation of alfalfa by Rhizobium meliloti. Cell 56:661-672.

Glenn, A. R., and Dilworth, M. J. 1981. The uptake and hydrolysis of disaccharides by fast- and slow-growing species of Rhizobium. Arch. Microbiol. 129:233-239.

Glenn, S. A., Gurich, N., Feeney, M. A., and González, J. E. 2007. The ExpR/Sin quorum-sensing system controls succinoglycan production in Sinorhizobium meliloti. J. Bacteriol. 189:7077-7088.

González, J. E., Reuhs, B. L., and Walker, G. C. 1996. Low molecular weight EPS II of Rhizobium meliloti allows nodule invasion in Medicago sativa. Proc. Natl. Acad. Sci. U.S.A. 93:8636-8641.

González, J. E., Semino, C. E., Wang, L.-X., Castellano-Torres, L. E., and Walker, G. C. 1998. Biosynthetic control of molecular weight in the polymerization of the octasaccharide subunits of succinoglycan, a symbiotically important exopolysaccharide of Rhizobium meliloti. Proc. Natl. Acad. Sci. U.S.A. 95:13477-13482.

Gosselin, I., Wattraint, O., Riboul, D., Barbotin, J., and Portais, J. 2001. A deeper investigation on carbohydrate cycling in Sinorhizobium meliloti. FEBS (Fed. Eur. Biochem. Soc.) Lett:45-49.

Hanahan, D. 1983. Studies on transformation of Escherichia coli with plasmids. J. Mol. Biol. 166:557-570.

He, F., Nair, G. R., Soto, C. S., Chang, Y., Hsu, L., Ronzone, E., DeGrado, W. F., and Binns, A. N. 2009. Molecular basis of ChvE function in sugar binding, sugar utilization, and virulence in Agrobacterium tumefaciens. J. Bacteriol. 191:5802-5813.

Hellweg, C., Pühler, A., and Weidner, S. 2009. The time course of the transcriptomic response of Sinorhizobium meliloti 1021 following a shift to acidic pH. BMC Microbiol. 9:37.
Her, G. R., Glazebrook, J., Walker, G. C., and Reinhold, V. N. 1990. Structural studies of a novel exopolysaccharide produced by a mutant of Rhizobium meliloti strain Rm 1021. Carbohydr. Res. 198:305-312.

Herridge, D., Peoples, M., and Boddey, R. 2008. Global inputs of biological nitrogen fixation in agricultural systems. Plant Soil 311:1-18.

Hoang, H. H., Gurich, N., and González, J. E. 2008. Regulation of motility by the ExpR/Sin quorum-sensing system in Sinorhizobium meliloti. J. Bacteriol. 190:861-871.

House, B. L., Mortimer, M. W., and Kahn, M. L. 2004. New recombination methods for Sinorhizobium meliloti genetics. Appl. Environ. Microbiol. 70:2806-2815.

Jacob, A. I., Adhamn, S. A. I., Capstick, D. S., Clark, S. R. D., Spence, T., and Charles, T. C. 2008. Mutational analysis of the Sinorhizobium meliloti short-chain dehydrogenase/reductase family reveals substantial contribution to symbiosis and catabolic diversity. Mol. Plant-Microbe Interact. 21:979-989.

Jensen, J. B., Ampomah, O. Y., Darrah, R., Peters, N. K., and Bhuvaneswari, T. V. 2005. Role of trehalose transport and utilization in Sinorhizobium meliloti-alfalfa interactions. Mol. Plant-Microbe Interact. 18:694-702.

Jiménez-Zurdo, J. I., van Dillewijn, P., Soto, M. J., de Filipe, M. R., Olivares, J., and Toro, N. 1995. Characterization of a Rhizobium meliloti proline dehydrogenase mutant altered in nodulation efficiency and competitiveness on alfalfa roots. Mol. Plant-Microbe Interact. 8:492-498.

Jones, J. D. G., and Gutterson, N. 1987. An efficient mobilizable cosmid vector and its use in rapid marker exchange in Pseudomonas fluorescens strain HV37a. Gene 61:299-306.

Jones, K. M. 2012. Increased production of the exopolysaccharide succinoglycan enhances Sinorhizobium meliloti 1021 symbiosis with the host plant Medicago truncatula. J. Bacteriol. 194:4322-4331.

Jones, K. M., Kobayashi, H., Davies, B. W., Taga, M. E., and Walker, G. C. 2007. How rhizobial symbionts invade plants: the SinorhizobiumMedicago model. Nat. Rev. Microbiol. 5:619-633.

Jones, K. M., Sharopova, N., Lohar, D. P., Zhang, J. Q., VandenBosch, K. A., and Walker, G. C. 2008. Differential response of the plant Medicago truncatula to its symbiont Sinorhizobium meliloti or an exopolysaccharide-deficient mutant. Proc. Natl. Acad. Sci. U.S.A. 105:704-709.

Kohler, P. R. A., Zheng, J. Y., Schoffers, E., and Rossbach, S. 2010. Inositol catabolism, a key pathway in Sinorhizobium meliloti for competitive host nodulation. Appl. Environ. Microbiol. 76:7972-7980.

Krol, E., and Becker, A. 2004. Global transcriptional analysis of the phosphate starvation response in Sinorhizobium meliloti strains 1021 and 2011. Mol. Gen. Genet. 272:1-17.

Latner, A. L., and Skillen, A. W. 1968. Isozymes in biology and medicine. Academic Press, New York.

Lehman, A. P., and Long, S. R. 2013. Exopolysaccharides from Sinorhizobium meliloti can protect against $\mathrm{H}_{2} \mathrm{O}_{2}$-dependent damage. J. Bacteriol. 195:5362-5369.

Leigh, J. A., and Walker, G. C. 1994. Exopolysaccharides of Rhizobium: synthesis, regulation and symbiotic function. Trends Genet. 10:63-67.

Leigh, J. A., Signer, E. R., and Walker, G. C. 1985. Exopolysaccharidedeficient mutants of Rhizobium meliloti that form ineffective nodules. Proc. Natl. Acad. Sci. U.S.A. 82:6231-6235.

Li, L., Jia, Y., Hou, Q., Charles, T. C., Nester, E. W., and Pan, S. Q. 2002. A global pH sensor: Agrobacterium sensor protein ChvG regulates acid-inducible genes on its two chromosomes and Ti plasmid. Proc. Natl. Acad. Sci. U.S.A. 99:12369-12374

Lu, H.-Y., Luo, L., Yang, M.-H., and Cheng, H.-P. 2012. Sinorhizobium meliloti ExoR is the target of periplasmic proteolysis. J. Bacteriol. 194:4029-4040.

Marketon, M. M., Glenn, S. A., Eberhard, A., and González, J. E. 2003. Quorum sensing controls exopolysaccharide production in Sinorhizobium meliloti. J. Bacteriol. 185:325-331.

Marroquí, S., Zorreguieta, A., Santamaría, C., Temprano, F., Soberón, M., Megias, M., and Downie, J. A. 2001. Enhanced symbiotic performance by Rhizobium tropici glycogen synthase mutants. J. Bacteriol. 183:854864.

Mendrygal, K. E., and González, J. E. 2000. Environmental regulation of exopolysaccharide production in Sinorhizobium meliloti. J. Bacteriol. 182:599-606.

Miller-Williams, M., Loewen, P. C., and Oresnik, I. J. 2006. Isolation of salt-sensitive mutants of Sinorhizobium meliloti strain Rm1021. Microbiology 152:2049-2052.

Mulligan, J. T., and Long, S. R. 1989. A family of activator genes regulates expression of Rhizobium meliloti nodulation genes. Genetics 122:7-18.

Oldroyd, G. E., Murray, J. D., Poole, P. S., and Downie, J. A. 2011. The rules of engagement in the legume-rhizobial symbiosis. Annu. Rev. Genet. 45:119-144.

Oresnik, I. J., Charles, T. C., and Finan, T. M. 1994. Second site mutations specifically suppress the $\mathrm{Fix}^{-}$phenotype of Rhizobium meliloti $n d v F$ mutations on alfalfa: Identification of a conditional $n d v F$-dependent 
mucoid colony phenotype. Genetics 136:1233-1243.

Oresnik, I. J., Pacarynuk, L. A., O’Brien, S. A. P., Yost, C. K., and Hynes, M. F. 1998. Plasmid encoded catabolic genes in Rhizobium leguminosarum bv. trifolii: Evidence for a plant-inducible rhamnose locus involved in competition for nodulation. Mol. Plant-Microbe Interact. 11: 1175-1185.

Pellock, B. J., Cheng, H. P., and Walker, G. C. 2000. Alfalfa root nodule invasion efficiency is dependent on Sinorhizobium meliloti polysaccharides. J. Bacteriol. 182:4310-4318.

Pellock, B. J., Teplitski, M., Boinay, R. P., Bauer, W. D., and Walker, G. C. 2002. A LuxR homolog controls production of symbiotically active extracellular polysaccharide II by Sinorhizobium meliloti. J. Bacteriol. 184:5067-5076.

Pickering, B. S., and Oresnik, I. J. 2008. Formate-dependent autotrophic growth in S. meliloti. J. Bacteriol. 190:6409-6418.

Pierre, O., Engler, G., Hopkins, J., Brau, F., Boncompagni, E., and Hérouart, D. 2013. Peribacteroid space acidification: A marker of mature bacteroid functioning in Medicago truncatula nodules. Plant Cell Environ. 36:2059-2070.

Poysti, N. J., Loewen, E. D., Wang, Z., and Oresnik, I. J. 2007. Sinorhizobium meliloti pSymB carries genes necessary for arabinose transport and catabolism. Microbiology 153:727-736.

Reinhold, B. B., Chan, S. Y., Reuber, T. L., Marra, A., Walker, G. C., and Reinhold, V. N. 1994. Detailed structural characterization of succinoglycan, the major exopolysaccharide of Rhizobium meliloti Rm1021. J. Bacteriol. 176:1997-2002.

Reuber, T. L., and Walker, G. C. 1993. Biosynthesis of succinoglycan, a symbiotically important exopolysaccharide of Rhizobium meliloti. Cell 74:269-280

Richardson, J. S., Hynes, M. F., and Oresnik, I. J. 2004. A genetic locus necessary for rhamnose uptake and catabolism in Rhizobium leguminosarum bv. trifolii. J. Bacteriol. 186:8433-8442.

Rittig, M. G., Alvarez-Martinez, M.-T., Porte, F., Liautard, J.-P., and Rouot, B. 2001. Intracellular survival of Brucella spp. in human monocytes involves conventional uptake but special phagosomes. Infect. Immun. 69:3995-4006.

Rossbach, S., Mai, D. J., Carter, E. L., Sauviac, L., Capela, D., Bruand, C., and de Bruijn, F. J. 2008. Response of Sinorhizobium meliloti to elevated concentrations of cadmium and zinc. Appl. Environ. Microbiol. 74:4218-4221.

Sambrook, J., Fritsch, E. F., and Maniatis, T. A. 1989. Molecular Cloning Manual, 2nd ed. Cold Spring Harbor Laboratory Press, Cold Spring Harbor, NY, U.S.A.

Spaink, H. P. 2000. Root nodulation and infection factors produced by rhizobial bacteria. Ann. Rev. Microbiol. 54:257-288.

Triplett, E. W., and Sadowsky, M. 1992. Genetics of competition for nodulation of legumes. Annu Rev Micorbiol 46:399-428.

Vanderlinde, E. M., Hynes, M. F., and Yost, C. K. 2013. Homoserine catabolism by Rhizobium leguminosarum bv. viciae 3841 requires a plasmid-borne gene cluster that also affects competitiveness for nodulation. Environ. Microbiol. 16:205-217.

Vincent, J. M. 1970. A manual for the practical study of root-nodule bacteria. Blackwell Scientific Publications, Oxford, England.

Watanabe, S., Kodak, T., and Makino, K. 2006. Cloning, expression, and characterization of bacterial L-arabinose 1-dehydrogenase involved in an alternative pathway of L-arabinose metabolism. J. Biol. Chem. 281:2612-2623.

Wu, C.-F., Lin, J.-S., Shaw, G.-C., and Lai, E.-M. 2012. Acid-induced type VI secretion system is regulated by ExoR-ChvG/ChvI signaling cascade in Agrobacterium tumefaciens. PLoS Pathog. 8:e1002938. Published online.

Yao, S.-Y., Luo, L., Har, K. J., Becker, A., Rüberg, S., Yu, G.-Q., Zhu, J.B., and Cheng, H.-P. 2004. Sinorhizobium meliloti ExoR and ExoS proteins regulate both succinoglycan and flagellum production. J. Bacteriol. 186:6042-6049.

Yost, C. K., Rath, A. M., Noel, T. C., and Hynes, M. F. 2006. Characterization of genes involved in erythritol catabolism in Rhizobium leguminosarum bv. viciae. Microbiology 152:2061-2074.

Yurgel, S. N., Rice, J., and Kahn, M. L. 2013. Transcriptome analysis of the role of GlnD/GlnBK in nitrogen stress adaptation by Sinorhizobium meliloti Rm1021. PloS one 8:e58028. Published online.

Zhao, J., and Binns, A. N. 2011. Characterization of the mmsAB-araD1 ( gguABC) Genes of Agrobacterium tumefaciens. J. Bacteriol. 193:65866596. 